\title{
Las Redes Sociales y la Nube: un nuevo Paradigma para los Procesos de Negocio
}

\author{
Mario Peralta, Carlos Salgado, Germán Montejano, Daniel Riesco \\ [mperalta, csalgado, gmonte, driesco]@unsl.edu.ar \\ Facultad de Ciencias Físico, Matemáticas y Naturales. Universidad Nacional de San Luis. \\ Ejército de los Andes 950. C.P.: 5700. San Luis, Argentina
}

DOI: 10.17013/risti.18.66-82

\begin{abstract}
Resumen: Desde la perspectiva de la complejidad de los procesos de negocio, una de las tecnologías más significativas para soportar su automatización son los Sistemas de Gestión Workflow. Para favorecer y flexibilizar dichos sistemas, es fundamental tener herramientas para medir su calidad. Debido a la globalización de la información y la comunicación, las empresas están migrando a la administración de sus procesos en la nube y a la utilización de las nuevas tecnologías. Las redes sociales permiten a las empresas tener una mejor disponibilidad de información. Esto presupone un nuevo paradigma de negocio. Se propone la aplicación de un framework para la evaluación y medición de procesos workflow en la evaluación de procesos workflow en la nube. En este contexto, se analiza el efecto de la utilización de las redes sociales en los procesos de Marketing y Comunicación de las organizaciones.
\end{abstract}

Palabras-clave: Workflow; Proceso de Negocio; Métricas; Cloud Computing; Redes Sociales; BPM.

\section{Social Networks and the Cloud: A New Paradigm for Business Processes}

Abstract: From business processes complexity perspective, one of the technologies that most significantly support its automation is Workflow Management Systems. Having a tool to measure the quality of these systems is essential. Due to the globalization of information and communication, companies are looking towards the management of processes on the cloud and the use of new technologies. The social networks allow the companies to have a better availability of information. This presupposes a new business paradigm. This paper proposes the application of a framework for evaluation and measurement of workflow processes to evaluate workflow processes in the cloud. In this context, the effect of the use of social networks in the process of marketing and communications organizations is analyzed.

Keywords: Workflow; Workflow Management Systems; Business Process; Metrics; Cloud Computing; Social Networks; BPM. 


\section{Introducción}

Analizando el ciclo de vida de los Procesos de Negocio (PN) (Georgakopoulos \& Tsalgatidou, 1998), es de gran importancia llevar a cabo una mejora continua de los mismos. Ello ha llevado a las organizaciones a buscar herramientas que proporcionen el soporte necesario para poder realizar dichas mejoras. Hoy en día, la Gestión de Procesos de Negocio (BPM, por si sigla del inglés Business Process Management) proporciona este soporte mediante los Sistemas de Gestión de Procesos de Negocio (BPMS, por si sigla del inglés Business Process Management Systems).

Una de las tecnologías más significativas para dar soporte a la BPM, son los Sistemas de Gestión Workflow (WMS, por si sigla del inglés Workflow Management Systems) que dan soporte a la automatización de los PN. Todo WMS debe garantizar que la organización realiza las tareas correctas, en el momento y de la forma adecuada. Por ello se considera fundamental tener una buena representación del proceso en la que se incluyan todos los aspectos de interés para el mismo que, además, permita adaptar esa representación a los cambios continuos que los negocios de hoy experimentan.

Otro de los aspectos fundamentales en todo proceso workflow, es tener medios que permitan medir sus elementos más relevantes con el fin de detectar las áreas y aspectos a mejorar del proceso y, de esta manera, promover su mejora continua. Para ello, es necesario proveer un marco que permita realizar dicha medición. De acuerdo a esta necesidad, siguiendo la metodología propuesta en (Serrano et al., 2002), se definió un conjunto de métricas elementales que sirven como indicadores de la complejidad estructural de los modelos de procesos workflow (Narayan Debnath et al., 2011; N. Debnath et al., 2012).

Desde otra perspectiva, debido a la globalización de la información y la comunicación, las empresas están dirigiendo su mirada a la administración de sus procesos en la nube. Desde el punto de vista de la administración de los procesos, un workflow puede ser controlado de forma manual, informatizada, o como una combinación de ambos métodos. Un esquema workflow permite a las empresas organizar las tareas y recursos a través de reglas que facilitan el control de los PN de la empresa. Es decir que, con un proceso workflow se logra un control total y absoluto de todas las tareas.

En este sentido, como se menciona en (Prieto et al., 2014) los workflows para procesos administrativos son utilizados en empresas e instituciones públicas pero, para poder utilizarlos adecuadamente en sus distintas áreas y departamentos, deben ser adaptados a las características propias de cada uno de ellos, respetando las normas que regulan el proceso a nivel general. En este sentido, las empresas interesadas en llevar su negocio a la nube, necesitan mantener ese control. Por ello, es fundamental que los procesos workflow sean de alta calidad.

Desde este punto de vista, un medio para obtener procesos de alta calidad, fácilmente mantenibles y adaptables, es proveer modelos de calidad de dichos procesos. Esto es válido para todo proceso workflow independientemente de dónde esté alojado. En particular, si los procesos serán administrados en la nube, será de gran utilidad tener herramientas que permitan evaluar la calidad de dichos procesos. 
Dentro de dicha globalización, la virtualización de las comunicaciones y la información ha permitido que las redes sociales convencionales sean más accesibles a todo el mundo, permitiendo a los seres humanos comunicarse de una manera global y dinámica (Coz Fernández et al., 2012). Esta manera de comunicarse se ha generalizado en su uso de manera tal que las empresas no pueden dejar de incorporarlas en sus procesos de comunicación.

Bajo estas consideraciones, en este trabajo presentamos los resultados de aplicar el marco de trabajo propuesto en (Narayan Debnath et al., 2015) para evaluar el impacto de las redes sociales en los procesos de comunicación de todo proceso de negocio en la nube.

El resto del trabajo se organiza como sigue. En la sección 2 se presenta una breve descripción de los trabajos relacionados. La sección 3 se delinea la influencia de las redes sociales en la administración de procesos workflow en la nube y se presentan las métricas utilizadas para llevar a cabo la medición de dicha influencia. La sección 4 describe la aplicación de las métricas propuestas en la toma de decisión sobre qué procesos migrar a la nube. En la sección 5 se describe la aplicación de nuevas métricas que permiten medir la influencia de la utilización de las redes sociales en los procesos de comunicación de la organización. Finalmente, en la sección 6 se presentan las conclusiones y trabajos futuros.

\section{Trabajos Relacionados}

En el campo de los procesos workflow, se puede observar un importante trabajo en cuanto al modelado de dichos procesos, surgiendo así diversas líneas de investigación, como el uso de Patrones Workflow para realizar la evaluación y/o comparación de distintos lenguajes de modelado, (van der Aalst et al., 2002; White, 2004; Petia Wohed et al., 2004; Petia Wohed et al., 2002; Petia Wohed et al., 2006). Desde otro punto de vista, en el ámbito de la medición, se pueden observar diversos trabajos en cuanto a la medición de los PN como en (Rolon et al., 2006; Rolon et al., 2005). Sin embargo, y a pesar de la importancia de medir la calidad de los procesos workflow, es muy poco el trabajo detectado en cuanto a la medición de calidad de estos procesos y de los modelos que los representan. Entre los trabajos en este campo se puede destacar las propuestas realizadas en (Cardoso, 2005a, 2005b, 2006), donde se propone una métrica para la medición de la complejidad del flujo de control basada en la complejidad ciclomática de McCabe. Otros trabajos que se pueden mencionar en este campo es la propuesta introducida en (Hajo A. Reijers, 2003; Hajo A. Reijers \& Vanderfeesten, 2004). En estos trabajos los autores introducen métricas para evaluar la cohesión interna de las actividades en un proceso workflow y el acoplamiento entre sus actividades. Estas propuestas presentan alternativas para la medición de algunos aspectos del modelado de procesos workflow.

En (Lee et al, 2015) los autores presentan el algoritmo “Reducción Efectiva Máxima (MER: Maximun Effective Reduction)", una solución eficiente que optimiza el uso de los recursos de un workflow planificado generado por cualquier algoritmo de planificación particular.

Desde otro punto de vista, en (Saleem et al., 2014) se propone un framework que permite generar conjuntos múltiples de workflows compatibles con múltiples organizaciones que colaboran entre sí. El framework propuesto apoya la promulgación y colaboración en 
tiempo de ejecución de los workflows generados. Este framework permite a los usuarios ahorrar tiempo y recursos que de otra manera se gastaría en el modelado, la conciliación y la reingeniería de los workfows.

Respecto de Cloud Computing, en la actualidad existen diversas herramientas y frameworks que permiten trabajar los procesos workflow en la nube. En este campo, esta investigación radica en la adaptación del marco de trabajo propuesto para su utilización en la evaluación de procesos workflow en la nube. Para ello, se proponen nuevas métricas que ayuden a medir las distintas características de los procesos workflow en la nube. El objetivo de dichas métricas es brindar una medición de la complejidad estructural de los procesos workflow y la relación de dichos procesos con su entorno. En este contexto, se aplicaron las métricas propuestas en la evaluación de los procesos workflow de una empresa del medio, la cual pretende migrar a la nube sus procesos con el fin de incrementar su competitividad (Narayan Debnath et al., 2015).

Con la aplicación de las métricas se pretende mostrar y determinar en qué grado el modelado de procesos workflow ayuda en la mejora continua de los procesos de negocio que se quieren llevar a la nube. Esto ayudará a proveer un medio para lograr procesos que sean más fácilmente mantenibles a partir de su entendibilidad y modificabilidad. Estas son propiedades muy importantes que deben tener los sistemas workflow actuales debido al dinamismo de los PN que ellos gestionan y que obligan al cambio y adaptación continua de estos sistemas. Más aún si se encuentran en la nube.

Los seres humanos, por su naturaleza, son sociables, y la cotidianidad en el uso de internet ha provocado que exporten sus usos y costumbres desde un "mundo real" a un "mundo virtual" (Coz Fernández et al., 2012). El trabajar en la nube lleva a tener que diseñar estrategias de marketing en internet. Hoy en día el mundo digital no deja de ofrecer nuevas herramientas y soluciones, para incrementar la efectividad de muchas de las técnicas usadas para la captación de nuevos clientes. Una de estas mejoras en los últimos años ha sido la posibilidad de automatizar muchas acciones de marketing para acortar tiempos de espera y así aumentar la satisfacción de los usuarios que interactúan con una página web. Una herramienta que permite esta automatización es el uso de las diversas redes sociales existentes en la actualidad. Estas redes ayudan en gran medida a fidelizar a los clientes.

Desde otro punto de vista, la tendencia actual va de la mano de los dispositivos móviles y la ubicuidad, y abre la posibilidad de numerosas nuevas alternativas como la generación de compras más "de contexto", y de brindar soluciones a la medida y la personalización del consumidor, desde su ubicación geográfica, preferencias e historial de compra, etc.

Los internautas que realizan compras a través de un dispositivo móvil, prefieren cada vez más utilizar una aplicación en vez de un navegador, por comodidad y velocidad. Entre los beneficios de comprar mediante una aplicación se pueden citar la confirmación instantánea del pago, recordatorios para aplicar ofertas y descuentos, y un registro digital de las compras. Todo ello resulta en que las compras a través de teléfonos inteligentes y tabletas sean más fáciles que nunca.

Como se mencionó previamente, la virtualización de las comunicaciones y la información ha permitido que las redes sociales convencionales sean más accesibles a todo el mundo, 
permitiendo a los seres humanos comunicarse de una manera global y dinámica (Coz Fernández et al., 2012). Desde esta perspectiva, las organizaciones actuales se plantean el interrogante de si el uso de estas redes sociales y aplicaciones para estos nuevos dispositivos serán rentables y/o productivas para el negocio, y si lo son, en qué grado lo serán. Así, será de gran utilidad la definición y aplicación de herramientas que permitan medir el grado en que estas nuevas tecnologías influyen en el desempeño de la organización.

En base a lo expuesto previamente, en este artículo se presenta una extensión del trabajo publicado en (Narayan Debnath et al., 2015), en dicha extensión se propone un conjunto de métricas para medir la influencia de las redes sociales y el uso de aplicaciones móviles en el desempeño de los PN de una organización y la mejora continua de dichos procesos.

\section{Medición de calidad de Procesos Workflow en la Nube y las Redes Sociales}

En todo proceso workflow, un aspecto fundamental es tener herramientas que permitan medir sus elementos más relevantes con el fin de detectar las áreas y aspectos a mejorar del proceso y, de esta manera, promover su mejora continua. Para ello es necesario proveer un marco que permita realizar dicha medición y tener criterios que ayuden a tomar esta decisión. De acuerdo a esta necesidad, siguiendo la metodología propuesta en (Serrano et al., 2002), se definió un conjunto de métricas iniciales y elementales para la medición de la calidad de modelos de procesos workflow desde el punto de vista de su mantenibilidad (Peralta et al., 2008). Dichas métricas servirán como indicadores de la complejidad estructural de los modelos de procesos workflow. Dichas métricas son una adaptación de las propuestas en (García, 2004) para la medición de procesos software. Las métricas propuestas ayudarán a la evaluación, comparación y mejora de los modelos workflow y, en consecuencia, de los procesos que ellos representan. En este contexto, se han realizado experimentos aplicando dichas métricas para la evaluación y comparación de lenguajes de modelado (Narayan Debnath et al., 2011; Peralta et al., 2007), como parte del proceso de su validación práctica. Además, se las aplicó en la evaluación de los modelos de proceso de una empresa del medio que, debido a las exigencias del mercado y a la fuerte tendencia de la globalización de los negocios, pretende subir sus procesos a la nube (Narayan Debnath et al., 2015).

Llevar el negocio a la nube brinda una nueva alternativa para poder ser competitivo en el mercado. Basado en ello, se busca mejorar las condiciones de trabajo y los costos, para poder brindarles a los clientes las mejores alternativas y servicios, tanto en tiempo como en calidad. En este contexto se adaptaron las métricas propuestas en (Narayan Debnath et al., 2011; Peralta et al., 2007) y se definieron nuevas métricas que permiten medir las características propias de los $\mathrm{PN}$ y aquellas inherentes a la nube, como por ejemplo, la comunicación entre procesos alojados en distintas nubes, la comunicación con otros procesos, entre otras.

Cloud Computing se puede pensar como un modelo de aprovisionamiento rápido de recursos de TI que potencia la prestación de servicios, negocioy TI, facilitando el trabajo del usuario final y del prestador del servicio. Describe un nuevo modelo para complementar, consumir y proveer servicios de TI basados en protocolos de Internet, y que por lo general consiste en el aprovisionamiento de recursos escalables dinámicamente. 
Acorde a ello, para lograr su objetivo, los proveedores de Cloud Computing proveen aplicaciones de negocio en línea que se acceden desde otro servicio Web o software como un navegador Web, mientras que el software y los datos se almacenan en los servidores. En este nuevo modelo de gestión de la información, los datos sensibles del negocio no residen en las instalaciones de las empresas, lo que podría generar un contexto de alta vulnerabilidad de la información. Desde el punto de vista de la BPM, las organizaciones empiezan a adaptarse a esta nueva tendencia y necesitan "subir su negocio a la nube". Sin embargo, debido a la estructura y las relaciones que se pueden dar en un PN, no siempre es posible llevar a la nube el negocio completo.

Esto lleva a las empresas a tener que realizar un estudio y análisis de sus procesos para determinar cuáles de ellos son factibles de llevar a la nube. Para ello es necesario contar con algún medio para poder realizar dicha evaluación. Bajo estas consideraciones, será de gran ayuda poder anticiparse a problemas o necesidades futuras. Para ello, es necesario complementar el estudio estructural de los procesos workflow con un análisis del rendimiento de esos procesos ejecutándose en la nube.

Para garantizar un buen rendimiento, es importante determinar la buena reputación comercial del proveedor como confiable, rápido, seguro y eficiente. Si falta alguna de estas métricas de rendimiento, el proveedor no tiene forma de chequear cuán bien se desempeña la aplicación en la nube. El mal rendimiento puede resultar en fallas inesperadas del servicio que dejan a los usuarios varados sin la información que necesitan para tomar decisiones comerciales. Para supervisar el rendimiento se puede configurar un tablero de métricas de rendimiento. Cuando una de las métricas muestra signos de inclinación hacia resultados negativos, debería poder accederse a las herramientas para identificar los posibles problemas de la aplicación antes que los usuarios tengan acceso a ellas. Algunas métricas de rendimiento disponibles, en distintos materiales bibliográficos, incluyen las siguientes (Myerson, 2013):

- Métricas de control de estado: se refieren a cuán bien responde la aplicación de forma correcta en los estados subsiguientes. Mientas la mayoría de las aplicaciones se encuentran inherentemente en control de estado, nunca se sabe cuándo se convierten en inestables.

- Métrica de control de versiones: hacen referencia a cuán bien un nuevo diseño evita romper las funciones de la aplicación existente, incluso si el estado de control previo de la aplicación respondió de forma correcta desde un estado a otro hasta que finalizan las tareas de la aplicación.

- Umbral de recursos: se refiere a cuán bien se equilibra el consumo de recursos de forma dinámica para las aplicaciones en la nube.

- El umbral del usuario: se refiere a cuán bien puede acceder un usuario de forma concurrente a la aplicación hasta el límite especificado en la licencia del usuario desde el proveedor.

- El umbral de solicitud de datos: se refiere a las solicitudes de datos que pueden procesarse rápidamente.

- El umbral de respuesta: se refiere a cuán rápido responde la aplicación a la solicitud de datos del usuario o a una parte de la aplicación para la otra parte. 
Algunas de estas métricas fueron aplicadas en (Peralta et al., 2015). A partir de dicho trabajo, las posibilidades para la aplicación y validación de dichas métricas se ampliaron en lo que respecta al marketing y fidelización de los clientes. En la actualidad existen muchas posibilidades de redes sociales y medios de comunicación, ya conocidos y utilizados por los clientes y proveedores de la organización.

El auge de las redes sociales y la necesidad imperiosa de las empresas de mantenerse competitiva lleva a que cada organización utilice los distintos canales de comunicación e intente aprovecharlos al máximo. El buen nombre y desempeño de las comunicaciones y status de la marca depende del aprovechamiento de las características de las redes sociales y que el negocio en su totalidad o en parte se encuentre en la nube.

Partiendo de estas premisas, teniendo una visión a futuro y previendo nuevos actores en lo que respecta a la interacción de los procesos de negocio, se comenzó a estudiar los beneficios del uso de redes sociales como Facebook, Twitter, Instagram, etc., como nuevos canales y herramientas de comunicación y transmisión entre la empresa y el mundo. Para realizar dicho estudio, se categorizaron a los clientes en tres subcategorías: Clientes Potenciales, Clientes de la empresa y Público visitante. Todos ellos pueden visitar la empresa a través de: Sitios Web, Redes Sociales, Correos Electrónico. Siempre pensando a la empresa a disposición para satisfacer los requerimientos de sus clientes y proveedores.

Para lograr este objetivo, es necesario medir, analizar, estimar y proyectar los procesos de marketing, comunicación, etc. Para ello, se incorporan al marco propuesto, métricas que miden distintas características de las redes sociales. Estas métricas se pueden dividir en:

- Métricas de actividad: Son las métricas que cuantifican el volumen de actividad que estamos desplegando en los canales sociales.

- Métricas de alcance: Son las métricas que permiten obtener información sobre el público, y su ritmo de crecimiento potencial y efectivo.

- Métricas de compromiso: Las métricas de engagement o "compromiso" Tratan de poner en cifras a la parte de la audiencia que interactúa con tus contenidos, mostrando un interés real y compartiendo de forma asidua.

- Métricas de adquisición: Con ellas se empieza a medir una parte de la audiencia que ha establecido una relación más profunda con la marca, empresa u organización. Por esa razón, las métricas están normalmente vinculadas con la página web oficial de la marca, y las conexiones entre esta y los canales sociales.

- Métricas de conversión: Aportan datos acerca de aquellos leads que han realizado alguna acción deseada (como una venta, por poner un ejemplo obvio).

- Métricas de fidelización: Su función es la de aportar información sobre los leads o clientes que regresan tras haber realizado una conversión.

Estas métricas se han incorporado al conjunto de métricas propuestas en (Narayan Debnath et al., 2015), para complementar el estudio de los modelos en las primeras fases de los proyectos, apoyando con información referente a los distintos servicios que brindan los proveedores donde serán luego implantados los PN. Esta información de performance sirve para hacer estimaciones, simulaciones y demás tareas para la toma de decisiones acerca de qué parte del negocio y en qué tiempo se traslada a la nube. 
Continuando con esta línea de investigación, con el objetivo de validar las nuevas métricas, las mismas se aplicaron en el contexto de la organización bajo estudio. En este sentido, la figura 1 muestra el ámbito de aplicación de las métricas en el contexto mencionado.

\section{Aplicación de Métricas para la Evaluación de Procesos Workflow en la Nube de una Empresa del Medio}

Acorde a lo expresado en las secciones previas, se aplicaron las métricas propuestas en la evaluación de los procesos workflow de una empresa del medio. En este sentido, los procesos en los que la empresa mostró un particular interés, en el inicio del proyecto, fueron los procesos denominados: “Compras y Pagos” “Ventas y cobranzas”. La elección de ambos procesos se debió a que presentan una fuerte interacción con diversos actores, internos y externos a la empresa. Dichos procesos se encontraban semi-automatizado.

Desde este punto de vista, las tareas automatizadas se implantaron en un servidor de aplicaciones en la nube. En cuanto a las tareas no automatizadas, corresponden a la comunicación entre los participantes de los procesos, las cuales se realizaban vía telefónica, o a través de un servicio de mensajería. Al subir los procesos a la nube, se optó por contratar un servicio de correo electrónico corporativo, ya que la empresa no tenía implementado dicho servicio.

A partir de esta situación, se debía evaluar el costo en la comunicación entre las actividades implementadas en las distintas nubes. Desde este punto de vista, las métricas propuestas son válidas para la medición de la complejidad estructural de un modelo de procesos, y por consiguiente del proceso que dicho modelo representa. Sin embargo, era necesario medir la complejidad de comunicación entre actividades de distintos procesos instalados en distintas nubes. Por ello, se definieron nuevas métricas que permiten medir dicha complejidad desde el punto de vista de la comunicación entre las actividades de las distintas nubes, del número de actividades por nube, proporción del número de actividades por nube, etc. Por ejemplo, dos de dichas métricas son NDC(n): Número de dependencias de la nube n con otras nubes; y DDC(n1, n2): Grado de dependencia entre las nubes n1 y n2 (Narayan Debnath et al., 2015).

Para obtener una mejor valoración de la conveniencia de migrar los procesos o subprocesos y determinar qué procesos o actividades de un proceso migrar, se aplicó el marco propuesto. En este sentido, la valoración de calidad del proceso ya implantado sirvió como un indicador para determinar la conveniencia de realizar la migración de dicho proceso a la nube.

Para lograr objetivo planteado, y como lo establece el marco de trabajo propuesto, en primer lugar se estableció cuáles de las métricas de modelos de procesos workflow propuestas en el mismo, (Narayan Debnath et al., 2011; N. Debnath et al., 2012), podrían capturar datos e información valiosa para el análisis de los procesos y la posterior toma de decisiones.

Una vez determinadas las métricas a aplicar, el análisis de los modelos comenzó con la tabulación de la aplicación de las métricas a dichos modelos. Luego, se analizó si esa 


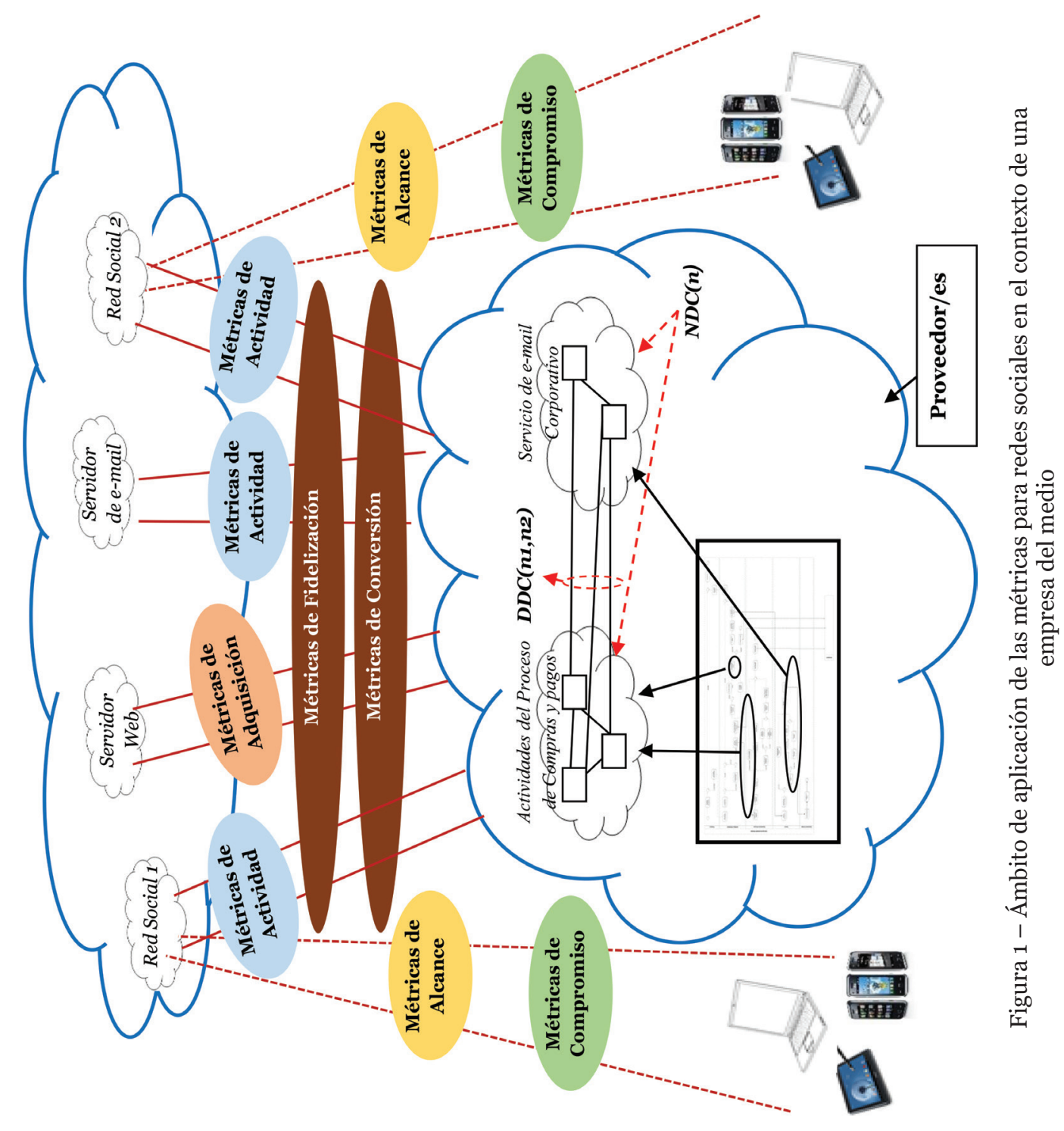


medida era suficiente o era necesario definir nuevas métricas, o adaptar las ya definidas para el nuevo modelo de negocio.

Una medida interesante, es la proporcionada por la métrica NDepPrA (mw), que indica el Número total de dependencias entre actividades de un modelo. Esta métrica, aporta información acerca de qué actividades son necesarias que terminen para poder continuar con la siguiente actividad del proceso. Sin embargo, además de contabilizar las dependencias que preceden a cada actividad, es necesario poder identificar si esa actividad se encuentra en el mismo servidor y/o proveedor, es decir en la misma nube, ya que ahora no sólo se debería saber qué actividades deberían terminar sino el tiempo de comunicación que podría influir en la consecución de la ejecución. Para ello, se propone la métrica NDepPrANUBE (mw) que, aplicada al modelo, indica el número de actividades que deben terminar en las distintas nubes para que puedan continuar las demás actividades. Esta medida es un valor crítico para la consecución del negocio. Por ello, es necesario prestar particular atención para que no se convierta en un cuello de botella (Peralta et al., 2015).

Desde otro punto de vista, se aplicó la métrica NDC, que establece el grado de comunicación de una nube con el resto. Esta medida mostró un valor elevado para ambas nubes, es decir que había una alto grado de comunicación con el resto de los procesos, ya sea que estuvieran o no alojados en la nube. Esta situación se vio más acentuada en la nube Correo Electrónico Corporativo, el cual es compartido con los procesos de los proveedores y con procesos de la empresa que aún no han sido subidos a la nube.

Desde otra perspectiva, surge el problema de la toma de decisión acerca de qué proveedor contratar. El marco de trabajo propuesto hace uso de las métricas de rendimiento, que permiten monitorear la performance de los servidores de los distintos proveedores que ofrecen los servicios. Para ello se definen indicadores haciendo uso de benchmarking y/o de la experiencia de los expertos del dominio. Estos indicadores se utilizaron para analizar, interpretar y decidir en cuanto a la calidad de servicio de la nube elegida.

Un atributo a medir consiste en la cantidad de usuarios que pueden acceder simultáneamente al servicio. Esta medida está dada por la cantidad de licencias adquiridas. Se considera un valor aceptable el 70\% de las licencias compradas, dejando un margen para evitar saturación de los servicios.

Otro atributo es el tiempo de respuesta. Para ello, se consideró hasta 4 segundos como un valor bueno. Por debajo de ese umbral es considerado muy bueno y excelente. Mientras que si lo supera degrada la respuesta de la aplicación y por consiguiente se hace perceptible para los clientes.

Es importante considerar la cantidad de recursos que consume cada nube y cómo se planifica su uso. Si la cantidad está por arriba del 50\% de los recursos de cada instancia empieza a degradar el funcionamiento por lo tanto se considera malo. Por debajo del 50\% es un valor bueno porque tiene disposición de recursos para satisfacer las demandas. En función de lo expresado, se tomaron indicadores que ayudaron a la gerencia de la empresa a decidir qué proveedor de servicios web contratar. En (Peralta et al., 2015) se puede encontrar en detalle el proceso de evaluación y aplicación de dichos indicadores y los resultados obtenidos en este punto. 


\section{Aplicación de Métricas para la Evaluación de los Procesos de Marketing y Comunicación de Procesos Workflow en la Nube}

En una nueva etapa del proceso de migración y mejora se deben adaptar los procesos de marketing y publicidad de la empresa a las nuevas tecnologías y herramientas de comunicación actuales, según se desprenda del análisis y evaluación de las nuevas posibilidades que brinda el mercado y dichas tecnologías. Algunas redes sociales como Facebook son de uso común por la mayoría de los actores que interactúan con las organizaciones. Para el estudio de la conveniencia de actualización, mejora y acondicionamiento de los procesos de marketing de la empresa, se aplicaron las métricas para redes sociales que forman parte del tablero de métricas. La situación a estudiar forma parte de la estrategia de negocio de la empresa motivo de estudio. Se procedió a comparar la distribución de datos e información, es decir publicidad y demás intereses de la organización en redes sociales y aplicar el método para la toma de decisiones.

Para lograr tal objetivo, se pueden usar métricas tales como la tasa de retorno que permite saber qué clientes regresan a la empresa. Dicha tasa es calculada como la diferencia entre los que no lo hacen, y los que sí. Dicha métrica queda definida

$$
\mathrm{TR}=\mathrm{N}^{\circ} \text { IPRegresan }-\mathrm{N}^{\circ} \text { IPNoRegresan }
$$

Esta métrica indirecta se calcula a partir de los datos que recolectan las métricas: $\mathrm{N}^{\circ}$ IPRegresan y $\mathrm{N}^{\circ}$ IPNoRegresan. Estos datos se obtienen a partir de una aplicación que contabiliza la cantidad de accesos por IP y registra la periodicidad de acceso de cada IP registrado. A partir de estos datos, se definieron indicadores para hacer una interpretación de la información recolectada. Uno de estos indicadores se definió de la siguiente manera:

\begin{tabular}{|c|c|c|c|}
\hline Más de $70 \%$ & Entre 61\% y 70\% & Entre $51 \%$ y $60 \%$ & Hasta $50 \%$ \\
\hline 1 & 0.75 & 0.4 & 0.2 \\
\hline
\end{tabular}

Para el contexto de trabajo de la organización, que los clientes que eligen sus productos regresen a hacer compras, pedidos o consultas en un 60\% o 70\% indica que el cliente se fideliza con la marca y servicios brindados. Que el número de retornos de visitantes sea menor al 50\%, pone de manifiesto que es necesario revisar o prestar atención al desempeño de las aplicaciones o herramientas de comunicación de la organización. Por ejemplo: en un principio, la tasa de retorno de los clientes fue inferior al 40\%. Eso fue un llamado de atención, los técnicos y encargados de la oficina de comunicación y difusión tuvieron que analizar los datos e información que se estaban presentando. En la actualidad, cada vez hay más personas que utilizan los teléfonos móviles para comparar precios, averiguar sobre una marca, empresa o producto, y concretar sus compras. Este crecimiento se ve impulsado por el incremento en el tamaño de las pantallas de los "smartphones", la optimización móvil de páginas web, el desarrollo de aplicaciones y la simplificación de los procesos de pagos móviles, etc.

De ese estudio surgió que había que hacer las aplicaciones y sitios responsive. Ya que las aplicaciones existentes en la empresa no se adaptaban fácilmente a las pantallas móviles. Estos son problemas o inconvenientes que introducen las nuevas tecnologías. Quizás parezcan obvios, pero en la mayoría de las veces son considerados triviales a la 
hora de la migración y son dejados de lado. Sin embargo, pueden conducir a un alto grado de rechazo por parte de los clientes, quienes dejarán de utilizarlos.

La satisfacción de los clientes es el objetivo principal de toda empresa. Este es uno de los puntos principales que se persigue en pos de una buena calidad de servicio. En la actualidad, el mundo de los negocios se encuentra en un camino hacia la personalización, hacia la individualización de cada uno de los consumidores 'online' por parte de las empresas. El objetivo de ellos es poder brindarles un servicio que sea adecuado a cada consumidor y, de esta manera, mejorar la conversión de cada uno de los negocios. Para obtener el Índice de satisfacción del cliente, se realizaron encuestas. Estas encuestas fueron divididas en conjuntos de preguntas con una puntuación entre o y 100. Las encuestas fueron llevadas a cabo a través de distintos canales: sitio web de la empresa, correo electrónico y en el interior de la empresa misma. Para trabajarlas se hicieron en la modalidad cerrada. Esto permitió un análisis automatizado y rápido. Haciendo el cálculo de estadísticos de centralización y dispersión.

Para interpretar estos resultados, se definió el indicador de satisfacción del cliente de la siguiente manera:

\begin{tabular}{|c|c|c|c|}
\hline $\begin{array}{c}\text { Más de 90 } \\
\text { puntos }\end{array}$ & $\begin{array}{c}\text { Entre 71 y 90 } \\
\text { puntos }\end{array}$ & $\begin{array}{c}\text { Entre 51 y 70 } \\
\text { puntos }\end{array}$ & $\begin{array}{c}\text { Menos de 50 } \\
\text { puntos }\end{array}$ \\
\hline 1 & 0.70 & 0.5 & 0.1 \\
\hline
\end{tabular}

En este punto se procedió a confrontar los valores obtenidos del análisis de las encuestas con el indicador de satisfacción. El resultado que arrojó la encuesta es que en promedio los clientes puntuaban su satisfacción con valores cercanos al 65\%. Si bien los valores no fueron del todo malos, fue un indicador de que algo en los cambios introducidos estaba molestando a los clientes. Para mejorar y permitir que los clientes se adaptaran al nuevo modelo de negocio de la empresa. Se va a esperar un tiempo prudencial de aprendizaje y adaptación de los clientes al nuevo modelo.

En una etapa posterior, aconsejados por el personal de estadística y administración, en una nueva fecha y espacio se repetirá la encuesta para averiguar si la visión de los usuarios sobre los nuevos medios de acceso a la empresa ha sufrido alguna modificación, dejando en claro que había una barrera a vencer, producto de las nuevas tecnologías y paradigmas de comunicación y aprendizaje; y a sabiendas que todo cambio trae aparejado cierta resistencia por diversos motivos como por ejemplo el acostumbramiento.

Además, se estudió las páginas vistas. Es decir, el número total de páginas que se han visto en un sitio web, con independencia del número y tipo de usuarios. Hay mucha afluencia a través de la web, se calculó un 33\%. Este porcentaje se estima que decaerá frente a las redes sociales, pero que hoy no se puede abandonar. El hecho de que muchos usuarios acceden por otros tipos de canales no significa que se deba descuidar la web. El indicador se definió utilizando el contador de acceso por página del sitio teniendo en cuenta el período de tiempo de permanencia en el sitio y recurrencia de los visitantes a cada página.

\begin{tabular}{|c|c|c|}
\hline Más de $50 \%$ & Entre 30 y $49 \%$ & Menos 29\% \\
\hline 1 & 0.50 & 0.25 \\
\hline
\end{tabular}


En la actualidad y con las posibilidades de las redes sociales, que la mitad o más de los clientes arriben a la empresa a través de la web se considera como un resultado excelente. Si se mantiene cerca del treinta por ciento y debajo de la mitad de los usuarios es aceptable dedicar recursos y tiempo al mantenimiento de los sitios web. Por debajo de esos niveles de visitas la influencia no es nula de ninguna manera, pero aporta poco peso a la toma de decisión.

Relacionado con la cantidad de clientes o usuarios de la empresa que acceden a través de Internet no se puede obviar la importancia e influencia de las redes sociales. Por lo tanto, se analizó y calculó el porcentaje de visitas sociales, es decir el total de visitantes que han llegado a la empresa a través de las redes sociales. Un total de 57\% accede a la empresa a través de estas redes. Mientras que el 10\% sigue usando los canales antiguos y que aún persisten como medios de comunicación e información. Se comparó con los Suscriptores por e-mail, es decir el número de personas que han dejado su correo electrónico suscribiéndose al blog corporativo. Además, se estimó el número de clientes potenciales obtenidos a través de las redes sociales. Bajo estas consideraciones, el indicador se definió de la siguiente manera:

\begin{tabular}{|c|c|c|c|}
\hline Más de $80 \%$ & Entre 50\% y 80\% & Entre 30\% y 49\% & Menos 29\% \\
\hline 1 & 0,75 & 0.50 & 0.25 \\
\hline
\end{tabular}

Cabe destacar que los indicadores y métricas descriptos son algunos de los que conforman el tablero de métricas e indicadores propuesto para el análisis y estudio de los procesos workflow. En este sentido, para realizar el informe que se eleva a la gerencia de la empresa, se aplicaron otras métricas como:

- Ratio de contenidos: las piezas totales de contenido que se han producido, en comparación con el período anterior.

- Ratio de publicaciones: el total de posts, actualizaciones y contenidos compartidos en relación al período anterior.

- Tiempo medio de respuesta: la media de tiempo que tarda alguien de la corporación en responder una consulta, pregunta, etc.

- Tasa de respuesta: la cantidad de comentarios, menciones, dudas y problemas que han recibido respuesta por parte de la marca durante el período contemplado.

- Tasa de crecimiento: ritmo al que crece o decrece tu audiencia. Se calcula dividiendo a los nuevos seguidores entre el número total que forman tu audiencia.

- Notoriedad de la marca: menciones de la marca en el segmento de tiempo seleccionado.

- Alcance de una publicación: el número de personas que han visto unos contenidos determinados al menos una vez.

No obstante, ello, la gerencia de la empresa, teniendo el informe generado en su poder, tomó la decisión de autorizar la partida monetaria para contratar los proveedores para la nube. Respecto de la comunicación y marketing con todos los tipos de usuarios y/o clientes, es prioridad para la gerencia. Por ello, y acorde a los resultados, se concluyó que la utilización de las redes sociales es indispensable para el buen funcionamiento 
de la organización tanto en lo publicitario como comunicacional. En función de ello, se asignará personal para la diagramación, uso y control de estas herramientas.

\section{Conclusiones}

Ante la globalización de las comunicaciones y el comercio electrónico, y la necesidad de las organizaciones de mantener su negocio altamente competitivo, las empresas están migrando sus procesos de negocio a la nube. Esto se debe a que estar en la nube significa movilidad, seguridad, escalabilidad y elasticidad. Es decir, poder dimensionar los servicios a lo que se precisa, incluso programando necesidades periódicas, despreocuparse de los equipos y las tecnologías, del mantenimientos y reparaciones, de la compra de equipos, todo lo cual es atendido por el proveedor del servicio. El único requisito es tener una conexión a Internet estable y de un ancho de banda suficiente.

Desde otra perspectiva, en la actualidad cada vez más las personas tienden a utilizar las redes sociales y el uso delas nuevas tecnologías, comola tecnología móvil, para la búsqueda de artículos y oportunidades de compra, servicios, etc. Por ello, las organizaciones tienden a incorporar a sus negocios estos nuevos medios de comunicación, en muchos casos adaptando los servicios que tienen. Esto trae aparejado nuevas situaciones con puntos de conflictos y fallas provenientes de un nuevo modelo de negocio. Ello implica que hay que atacar y solucionar estos nuevos inconvenientes a través de la propuesta de nuevos marcos de trabajo, técnicas, métodos, herramientas, etc. que permitan abordar estas nuevas situaciones de manera organizada y repetible.

No obstante las ventajas que proveen la nube y las nuevas tecnologías, no siempre es posible, o no siempre las organizaciones están dispuestas a subir todos sus procesos a la nube y adaptarse a los nuevos paradigmas de comunicación, ya que no confían en la seguridad de la misma o porque consideran que no les será rentable. Por ello, los dueños de los procesos necesitan tener un medio que les permita evaluar la conveniencia de subir todos o parte de sus procesos a la nube y, en caso de subir alguno de ellos, decidir cuál o cuáles de ellos subir. $\mathrm{Al}$ igual que decidir si incorporar el uso de las redes sociales y las nuevas tecnologías que pueden surgir o están surgiendo para mejorar sus procesos de comunicación y marketing.

En el contexto de la presente investigación, consistente en definir un marco de trabajo para medir la complejidad estructural de los modelos de procesos workflow y los procesos que ellos representan, se propone un tablero de métricas para tal objetivo. Además, en el contexto de los procesos workflow en la nube, se definieron nuevas métricas y se utilizaron las propuestas en distintas fuentes bibliográficas que permiten medir el grado de acoplamiento y cohesión entre los procesos en la nube. Para las métricas de rendimiento se definieron indicadores que permitieron confrontar distintas evaluaciones de los servicios que brindan los diversos proveedores en los que se estaba interesado para migrar el negocio a la nube. Este marco, además de utilizarse para elegir un proveedor para llevar a cabo la migración del negocio, puede servir también para controlar el funcionamiento de los procesos o sub-procesos ya migrados. Esto permitirá anticiparse a errores o demoras en los servicios y estar preparados para darles una respuesta a los clientes.

Este marco posibilita medir los procesos workflow en la nube. Se miden atributos tales como: cantidad de clientes accediendo, tiempos de respuesta, cantidad de recursos 
utilizados, acoplamiento, cohesión, cantidad de tareas / subprocesos por nube, etc. Todos estos valores permiten tener una medida de la calidad de los modelos, lo que posibilita trabajar en la mejora continua de los procesos que dichos modelos representan. También es necesario definir indicadores de calidad o benchmarking para poder comparar y mejorar cada uno de los procesos de las empresas u organizaciones. En este sentido, el análisis llevado a cabo permite tener información referente a la distribución de los procesos en la nube. Lo cual servirá de base para posteriores trabajos o mejoras de los mismos.

Por otro lado, y considerando la difusión de las redes sociales y las nuevas tecnologías móviles, se definieron e incorporaron nuevas métricas e indicadores que permiten medir el grado en que afectan el buen desempeño del negocio.

En este contexto, se aplicaron estas métricas para evaluar los procesos de una empresa del medio que se encuentra en la tarea de subir su negocio a la nube con el fin de mantenerse en un alto nivel competitivo. Al respecto, en el presente trabajo se exponen los resultados de la aplicación de las métricas para medir la influencia de las redes sociales en el proceso de marketing y comunicación de la empresa.

En la continuidad del trabajo, se aplicarán las métricas definidas a nuevos casos de estudio que lleven a una mejor validación práctica de las mismas. Además, se analizará la necesidad de definir nuevas métricas para evaluar otros aspectos de las tecnologías de la información y la comunicación ligada a los flujos de procesos de negocio y su incidencia en el tipo de mercado actual.

\section{Referencias}

Cardoso, J. (2005a). Control-flow Complexity Measurement of Processes and Weyuker's Properties.

Cardoso, J. (2005b). How to Measure the Control-flow Complexity of Web Processes and Workflows Workflow Handbook 2005.

Cardoso, J. (2006). Approaches to Compute Workflows Complexity. Paper presented at the Dagstuhl Seminar, The Role of Business Processes in Service Oriented Architectures, Dagstuhl, germany.

Coz Fernández, J. R., Fojón Chamorro, E., Heradio Gil, R., \& Cerrada Somolinos, J. A. (2012). Evaluación de la Privacidad de una Red Social Virtual. RISTI - Revista Ibérica de Sistemas e Tecnologias de Informação, 59-73. doi: 10.4304/risti.9.59-73

Debnath, N., Peralta, M., Salgado, C., Baigorria, L., Montejano, G., \& Riesco, D. (2015). Metrics and Performance Indicators to Evaluate Workflow Processes on the Cloud. Paper presented at the 12th ACS/IEEE International Conference on Computer Systems and Applications. AICCSA 2015.

Debnath, N., Peralta, M., Salgado, C., Montejano, G., Riesco, D., \& Berón, M. (2011). Metrics for Evaluation of Workflow Models: An Experiment for Validation. 20th International Conference on Software Engineering and Data Engineering (SEDE 2011), USA. 
Debnath, N., Peralta, M., Salgado, C., Montejano, G., Riesco, D., \& Berón, M. (2012). Metrics for Evaluation of Workflow Models: An Experiment for Validation. Journal of Computational Methods in Science and Engineering. , 12 (Sumplement 1).

García, F. Ó. (2004). FMESP: Marco de Trabajo Integrado para el Modelado y la Medición de los Procesos Software. Doctoral. Universidad de Castilla La Mancha. España.

Georgakopoulos, D., \& Tsalgatidou, A. (1998). Technology and Tools for Comprehensive Business Process Lifecycle Management,. Workflow Management Systems and Interoperability. Springer $V . p ., 324-365$.

Lee, Y. C., Han, H., Zomaya, A. Y., \& Yousif, M. (2015). Resource-efficient workflow scheduling in clouds. Knowledge-Based Systems, 153-162.

Myerson, J. M. (2013). Best Practices to Develop SLAS for Cloud Computing. IBM Systems Journal.

Peralta, M., García, F., Piattini, M., \& Uzal, R. (2007). Un experimento Comparativo de dos Lenguajes de Modelado Workflow: YAWL vs Diagramas de Actividad. 8th Argentinean Symposium on Software Engineering (ASSE 2007), 145-154.

Peralta, M., Garcia Rubio, F. Ó., Riesco, D., Salgado, C., \& Montejano, G. (2008). Un Conjunto de Medidas para la Evaluación de Modelos Workflow. Congreso Argentino de Ciencias de la Computación (CACIC'O8).

Peralta, M., Salgado, C., Baigorria, L., Montejano, G., \& Riesco, D. (2015). Procesos Workflow en la Nube: Una Propuesta para Evaluar su Migración. 3er. Congreso Nacional de Ingeniería de Software/Sistemas de Información - 2015.

Prieto, Á. E., \& Lozano-Tello, A. (2014). Adaptación de Workflows basada en Ontologías. RISTI - Revista Ibérica de Sistemas e Tecnologias de Informação, DOI: 10.17013/ risti.14.67-82

Reijers, H. A. (2003). A Cohesion Metric for the Definition of Activities in a Workflow Process. Eighth CAiSE/IFIP8.1 2003, 116-125.

Reijers, H. A., \& Vanderfeesten, I. T. P. (2004). Cohesion and Coupling Metrics for Workflow Process Design. BPM 2004, LNCS 3080, 290-305.

Rolon, E., Garcia Rubio, F. Ó., Ruiz, F., \& Piattini, M. (2006). Validating a Set of Measures for Business Process Models Usability and Maintainability.

Rolon, E., Ruiz, F., Garcia Rubio, F. Ó., \& Piattini, M. (2005). Aplicación de Métricas Software en la Evaluación de Modelos de Procesos de Negocio. Revista Electrónica de la Sociedad Chilena de Ciencia de la Computación.

Saleem, M., Chung, P. W. H., Shaheen, F., \& Dai, W. (2014). A cross organisation compatible workflows generation and execution framework. Knowledge-Based Systems, 1-14. 
Serrano, M., Piattini, M., Calero, C., Genero, M., \& Miranda, D. (2002). Un método para la definición de métricas de software. Paper presented at the 1er Workshop en Métodos de Investigación y Fundamentos filosóficos en Ingeniería del Software y Sistemas de Información (MIFISIS'2002),

van der Aalst, W. M. P., ter Hofstede, A. H. M., Kiepuszewski, B., \& Barros, A. P. (2002). Workflow Patterns: Queensland University of Technology, Brisbane.

White, S. A. (2004). Process Modeling Notations and Workflow Patterns. In L. Fischer (Ed.), Workflow Handbook 2004: Published in association with the Workflow Management Coalition (WfMC).

Wohed, P., van der Aalst, W. M. P., Dumas, M., H.M. ter Hofstede, A., \& Russell, N. (2004). Pattern-based Analysis of UML Activity Diagrams.

Wohed, P., van der Aalst, W. M. P., Dumas, M., \& ter Hofstede, A. H. M. (2002). Pattern Based Analysis of BPEL4WS: Queensland University of Technology, Brisbane.

Wohed, P., van der Aalst, W. M. P., Dumas, M., ter Hofstede, A. H. M., \& Russell, N. (2006). On the Suitability of BPMN for Business Process Modelling. 4th International Conference on Business Process Management (BPM 2006), LNCS., Vienna, Austria. 